Revue d'histoire du XIXe siècle

Société d'histoire de la révolution de 1848 et des

révolutions du XIXe siècle

$51 \mid 2015$

Atlantique ibérique et migrations après les indépendances américaines

\title{
Enjeux des politiques de nationalité dans le contexte de migrations post-impériales : le cas de
} l'Argentine, 1853-1931

Nationality Policy in the Context of Post-imperial Migration: the Case of

Argentina, 1853-1931

Nationalitätenpolitik im Kontext postimperialer Migration: der Fall Argentinien, 1853-1931

Pilar González Bernaldo de Quirós

\section{OpenEdition}

Journals

Édition électronique

URL : http://journals.openedition.org/rh19/4922

DOI : $10.4000 /$ rh 19.4922

ISSN : $1777-5329$

Éditeur

La Société de 1848

Édition imprimée

Date de publication : 1 décembre 2015

Pagination : 71-87

ISSN : 1265-1354

Référence électronique

Pilar González Bernaldo de Quirós, «Enjeux des politiques de nationalité dans le contexte de

migrations post-impériales : le cas de l'Argentine, 1853-1931 », Revue d'histoire du XIXe siècle [En ligne],

51 | 2015, mis en ligne le 01 décembre 2018, consulté le 19 avril 2019. URL : http://

journals.openedition.org/rh19/4922 ; DOI : 10.4000/rh19.4922 


\section{PILAR GONZÁLEZ BERNALDO DE QUIRÓS}

\section{Enjeux des politiques de nationalité dans le contexte de migrations post-impériales: le cas de l'Argentine, 1853-1931}

Les échanges transatlantiques ont joué un rôle important aussi bien dans la consolidation des empires que dans leur crise, et c'est également au sein de ces échanges qu'ont émergé les nouvelles nations comme États souverains de droit international ${ }^{1}$. Pourtant l'histoire politique, comme d'ailleurs l'histoire des migrations atlantiques, ont eu tendance à penser ces questions dans le cadre que les historiographies nationales avaient bâti au long du XIX siècle, tant pour analyser l'émergence des nouvelles nations que pour expliquer les mouvements massifs de populations européennes vers le «Nouveau Monde». De sorte qu'une brèche herméneutique vint dissocier l'histoire des Empires de celle des Nations, de la même manière que l'on a dissocié celle des mobilités dans l'espace impérial de celle des migrations de masse. Or nous le savons, les indépendances ne mettent fin ni aux flux des migrations libres au sein du territoire de l'ex-empire ni aux transferts de main d'œuvre esclave². Bien au contraire, elles ont plutôt eu tendance à les accroître et à les diversifier. Ces faits maintenant largement reconnus invitent à élargir le cadre spatiotemporel d'analyse des mouvements migratoires de masse et à s'interroger sur les liens entre l'expérience impériale et l'histoire des migrations postérieures.

Je propose ici d'introduire cette approche à partir de l'analyse d'une question apparemment marginale et "technique» : celle de la politique de nationalité. La nationalité sert à définir les droits des personnes en fonction du lien avec un État et relève de compétences souveraines de l'État. En Amérique ibérique cette question a été au cœur des négociations entre les États qui émergent de la crise impériale et entre ces derniers et leurs ex-métropoles. Figure juridique, elle constitue également un instrument politique de régulation des mobilités, dont les différents États n’ont pas hésité à faire usage ${ }^{3}$.

1. Cf. Jeremy Adelman, Sovereignty and revolution in the Iberian Atlantic, Princeton, Princeton University Press, 2006.

2. Cf. José C. Moya, "Migración africana y formación social en las Américas, 1500-2000", Revista de Indias, LXXII, 255, 2012, p. 321-348.

3. Patrick Weil a consacré un important ouvrage à démontrer la dimension politique du droit de la nationalité. Cf. Patrick Weil, Qu'est-ce qu'un Français? Histoire de la nationalité française depuis la Révolution, Paris, Grasset, 2002. 
Nous aborderons cette question à partir du cas argentin, en faisant l'hypothèse que les politiques de nationalité, domaine de compétence exclusive de l'État souverain, répondent à des enjeux internationaux qui s'inscrivent dans une logique post-impériale où le plus global peut s'articuler avec le plus local. La fourchette chronologique choisie correspond à celle qui va de la promulgation de la Constitution de 1853 et l'introduction du principe de jus soli lors de son amendement en 1860 jusqu'à la promulgation du décret d'application de la loi de nationalité en 1931. Pour cette recherche j'ai principalement travaillé sur un corpus législatif et juridique.

\section{Peupler la nation en perpétuant UNE FIGURE JURIDIQUE IMPÉRIALE}

Les recherches sur la politique d'immigration en Argentine commencent souvent par un rappel de la sentence du juriste Juan Bautista Alberdi, auteur du texte qui inspire la Constitution Argentine de 1853-1860 : «En Amérique, gouverner c'est peupler " ${ }^{4}$. Le préambule de ce texte voté en 1853 donne le ton en déclarant que la Constitution est promulguée "pour nous, pour notre descendance et pour tous les hommes du monde qui veulent habiter le sol argentin». On ne saurait pas fixer un cadre plus large pour définir la communauté de destin. Certes, les arrières pensées racialistes ne sont pas absentes et pour Juan Bautista Alberdi le peuplement comme impératif de gouvernement ne saurait inclure les peuples "non civilisés", comme l'étaient à ses yeux les Chinois, les Indiens d'Asie et des Noirs d'Afrique. Mais ces préjugés ne se sont pas traduits par un texte législatif et l'Argentine est un des rares pays Américains à ne pas introduire le principe d'une sélection ethnique dans la loí5. Il importe de préciser que lorsque la Constitution est promulguée en 1853, la riche province de Buenos Aires avait failli à l'Union en faisant sécession en 1852. Elle ne réintégra la Confédération que lorsque les réformes accordées par une convention préalable furent introduites dans le texte constitutionnel en 1860. La Constitution de la Nation Argentine de 1860 qui en résulte conserve ce préambule ainsi que les principaux articles destinés à promouvoir l'immigration européenne comme précepte constitutionnel.

Il s'agit d'un texte d'une étonnante ouverture. En effet, outre le préambule, les premiers articles de la Constitution de 1853 consacrés aux droits et garanties ratifiés par la réforme de 1860, précisent le contenu en accordant

4. Ceci mène certains spécialistes à classer l'Argentine comme pays d'immigration de peuplement par rapport aux pays dits d'immigration de travail. $C f$. Juan Bautista Alberdi, Bases y puntos de partida para la organización política de la República Argentina, Buenos Aires, Eudeba, (1 $1^{\text {re }}$ édition 1852), 1966.

5. Cf. «Páginas explicativas de Alberdi», Juan Bautista Alberdi, Bases..., op. cit. Pour une analyse comparée des politiques migratoires dans les Amériques, une étude exhaustive dans David Fitz Gerald, David Cook-Martin, Culling the Masses. The Democratic Origins of Racist Immigration Policy in the Americas, Cambridge, Harvard University Press, 2014, p. 299-332. 
aux étrangers les mêmes droits civils qu'aux nationaux. L'article 14 décline ainsi les droits des "habitants" : travailler, pétitionner, circuler, publier, user et disposer de la propriété, professer librement un culte, s'associer, apprendre et enseigner. Par son art. 16, la constitution fixe le principe de l'égalité de tous les habitants devant la loi et devant l'emploi (la seule distinction ne peut être que celle de l'aptitude) ${ }^{6}$. L'unique article qui parle d'étrangers le fait pour établir les conditions d'accès à la nationalité : deux années de résidence continue sur le territoire, délai qui peut être raccourci en cas de services accomplis au profit de la République. Par le biais du statut d'habitant la constitution établit ainsi un cadre juridique très précis et libéral en matière de droits pour les étrangers. L’objectif de cette extraordinaire libéralité est explicité par Juan Bautista Alberdi lui-même : il s'agit de répondre à la fois aux impératifs économiques en attirant des capitaux et des bras et aux présupposés civilisationnels de la nation argentine par le biais de l'accueil d'une population exclusivement européenne censée apporter la civilisation ${ }^{7}$. L'originalité du projet de Juan Bautista Alberdi réside dans le fait de concevoir la politique d'immigration en termes de droits accordés aux étrangers. Mais la proposition était suffisamment exceptionnelle pour qu'il ait été obligé d'expliquer les raisons de cette singularité, qu'on ne trouvait dans aucun texte constitutionnel des nations alors prises comme modèle ${ }^{8}$. La vraie bizarrerie consiste à mon sens dans la longévité de ce texte, surtout si l'on tient compte de la remarquable instabilité constitutionnelle en Amérique latine au XIXe siècle. Car des exemples de constitutions rédigées sous l'impulsion de grands élans libéraux, la région en fournit plusieurs, aussi prodigues qu'éphémères 9 . Or cette constitution restera en vigueur pendant un siècle; même par la suite, le principe d'égalité de droits civils entre nationaux et étrangers garantis par la constitution sera repris dans toutes les constitutions suivantes de l'Argentine jusqu’à nos jours ${ }^{10}$. Cette continuité, nous la trouvons également dans la

6. La propriété des habitants est protégée par l'article 17, le 18 leur garantit le recours d'habeas corpus et le 28 fixe le principe de prééminence des garanties constitutionnelles sur toute initiative législative.

7. Sur le rapport entre la politique d'immigration et la construction nationale, une analyse lumineuse dans Tulio Halperin Donghi, "¿Para qué la inmigración? Ideologia y política inmigratoria en la Argentina (1810-1914)", in El Espejo de la Historia. Problemas Argentinos y perspectivas latinoamericanas, Buenos Aires, Ed. Sudamericana, 1987, (1 édition 1976), p. 191-238.

8. «Dans la constitution d'un pays européen, ce chapitre serait en trop, serait même peut-être insensé, car il tendrait à attirer ce que plutôt il convenait d'éloigner. Voici la raison pour laquelle nos copistes ne le trouvent pas dans les textes constitutionnels de l'Europe». Juan Bautista Alberdi dit s'inspirer dans la Constitution de l'État de Californie de 1849, "pensée pour créer un grand peuple en peu d'années ", $c f$. Juan Bautista Alberdi, Bases..., op. cit., p. 49-52.

9. On raconte que la constitution libérale dite "de Rionegro" adoptée par la Colombie en 1863 inspire à Victor Hugo ce commentaire désabusé «ce doit être un pays peuplé d'anges!», $c f$. Jean Pierre Minaudier, Histoire de la Colombie, de la conquête à nos jours, Paris, L'Harmattan, 1992, p. 151.

10. La réforme constitutionnelle de 1949 proposée par de Juan D. Perón reprend cette figure d'habitant en introduisant certes, dans son article 20, une condition nouvelle pour pouvoir en bénéficier : celle d'être entré légalement dans le pays. La constitution de 1957, qui remplace celle de Perón, va abroger cette condition, reprenant le texte de 1853, cf. Arturo E. Sampay, Las Constituciones de la Argentina (1810-1972), Buenos Aires, Eudeba, 1975. 
législation sur l'immigration, qui complète en 1876 le dispositif général mis en place afin de faire de l'Argentine une destination attractive pour l'Europe en mouvement. La continuité dans cette tradition juridique n'empêche pas, bien entendu, que les gouvernements aient pu la contredire, soit par le vote de lois anticonstitutionnelles, soit par le biais de certaines pratiques administratives destinées à contrecarrer son application. Ainsi les lois de Résidence de 1902 et de Sécurité Sociale de 1910, qui accordent à l'exécutif le pouvoir d'expulser les étrangers considérés comme comportant un risque pour l'ordre public, impliquent une claire entorse au droit des habitants. Toutes les deux seront souvent décriées comme étant anticonstitutionnelles, mais seulement abrogées en 1958. Comme l'a bien souligné Fernando Devoto, il faut cependant distinguer la tradition juridique, clairement ouverte aux droits des étrangers, des politiques de fait ${ }^{11}$.

En quoi consiste au juste cette tradition juridique? Il s'agit de la perpétuation de la figure de l'habitant, à mi-chemin entre l'étranger de passage et le national ${ }^{12}$. Comme ce dernier, l'habitant jouit de droits civils, mais ceux-ci ne sont fondés ni sur la naissance - jus soli - ni sur l'hérédité - jus sanguinis -; ce sont les droits acquis du fait de la résidence - jus domicili - qui prévalent. Et le bénéfice de ces droits n'exige ni la condition de réciprocité, comme l'établit le Code civil français, pourtant souvent proclamé comme le modèle du code civil argentin, ni une préalable naturalisation ${ }^{13}$. La Constitution réserve cependant les droits politiques aux nationaux, alors qualifiés de citoyens, introduisant ainsi une assimilation entre citoyens et nationaux que la figure de l'habitant dément pourtant. Car les droits accordés aux habitants dépassent la dimension privée des rapports entre les personnes. D’une part parce que certains droits civils comme celui de s'associer, de pétitionner ou de publier sont en lien direct avec la pratique de la citoyenneté, et que les étrangers ne manqueront pas d'en faire usage ${ }^{14}$. Mais aussi, de manière plus statutaire, par la dimension civique que revêtait la résidence dès lors qu'elle

11. Fernando Devoto, «El revés de la trama: políticas migratorias y prácticas administrativas en la Argentina (1919-1949)", Desarrollo Económico, volume 41, n 162 (juillet-septembre 2001), p. 281-304.

12. Certes l'Argentine n'est pas le seul pays à introduire cette figure. La France la connaît également jusqu'à la loi de 1889 à travers le statut d' «admis à domicile». Mais à la différence de la France c'est un statut garanti par la constitution et qui ne requiert aucune procédure administrative et qui n'est pas soumis à une contrepartie, Patrick Weil, Qu'est-ce qu'un Français..., op. cit., chap. 1 et 2.

13. Dalmacio Vélez Sarfield, le rédacteur du code, justifie cette absence dans le texte qui accompagne la remise du projet à l'intention du Ministre de la Justice Eduardo Costa par le fait «que la différence entre étrangers et nationaux avait disparu en Argentine au moment où la Constitution garantissait les mêmes droits pour tous», $c f$. Dalmacio Velez Sarfield, "Nota de elevación del libro primero del Código Civil», Buenos Aires, 21 de junio de 1865 in Código Civil completo con las notas del codificador Vélez Sársfield, Buenos Aires, 1865. Le texte est approuvé en 1869 et rentre en vigueur le $1^{\text {er janvier } 1871 .}$

14. Cf. Pilar Gonzalez Bernaldo, "Etrangers à la nation, citoyens dans la cité : l'expérience politique des étrangers dans la ville de Buenos Aires pendant la deuxième moitié du XIX siècle», in Pilar Gonzalez Bernaldo, Manuela Martini, Marie-Louise Pelus-Kaplan [dir.], Étrangers et sociétés, Rennes, Presses universitaires de Rennes, p. 115-125. 
était reconnue comme source de droit ${ }^{15}$. C'est en tant qu'habitants d'une commune (vecinos) que la plupart des constitutions provinciales vont reconnaître aux résidents soumis à l'impôt le droit de vote passif et actif dans leur commune. Ici encore, on peut constater une remarquable continuité, même si les situations peuvent différer dans le temps entre États provinciaux. Si nous prenons le cas de la province de Buenos Aires, qui concentre pendant les deux siècles postérieurs à l'indépendance le plus grand nombre d'étrangers, nous constatons qu'entre la première constitution provinciale de 1854 et celle de 1994 actuellement en vigueur, les étrangers ont bénéficié du droit de vote actif et passif aux élections municipales pendant toute la période avec des conditions de ressources et de résidence qui peuvent varier entre trois mois pour la constitution de 1873 et deux ans pour la constitution de 1994 -, à l'exception des sept ans écoulées entre la promulgation de la constitution provinciale de 1949 et son abrogation en 1956.

La crispation autour de cette question dans le débat d'aujourd'hui en Europe, ne devrait pas nous faire oublier la variété de statuts et de pratiques sous lesquelles les étrangers ont pu exercer la citoyenneté malgré leur condition de non-nationaux tout au long des $\mathrm{XIX}^{\mathrm{e}}$ et $\mathrm{XX}^{\mathrm{e}}$ siècles. Dans le monde hispanique, cela renvoie explicitement au droit de vicinité qui, comme l'a remarquablement bien démontré Tamar Herzog, sert depuis le XVI e siècle d'assise à la définition de la condition de "naturel» [natural] et se fonde sur le fait social impliqué par la vie en communauté - interactions quotidiennes, engagement dans la vie de la communauté par le biais de la famille, de la propriété et de l'impôt $\mathrm{t}^{16}$. L'auteure démontre comment la catégorie de vecino, qui depuis le Moyen Age repose sur le domicile de droit romain, s'impose pour légitimer aussi bien les nouvelles installations des populations catholiques sur les territoires de la péninsule ibérique encore sous domination arabe que, par la suite, la conquête et la colonisation des Indes Occidentales. Elle va conduire dès le XVIe siècle à la consolidation de la doctrine sur la liberté de migrer qui sera bientôt défendue comme droit naturel. Omniprésente dans l'ordonnancement juridique de l'Empire - les institutions des corporations territoriales étaient constituées autour du statut de vicinité - cette catégorie est introduite dans le langage constitutionnel du monde hispanique par la Constitution de Cadix (1812) ${ }^{17}$. La continuité entre le statut de vicinité de l'Empire et celui de citoyenneté locale post-impériale explique le consensus

15. Je reprends ici la distinction d'Étienne Balibar entre citoyenneté comme statut juridique et citoyenneté sociale productrice de statut, $c f$. Etienne Balibar, Droit de cité, Paris, PUF, Quadrige, 2002, p. 53-61.

16. Cf. Tamar Harzog, Defining Nations. Immigrants and Citizens in Early Modern Spain and Spanish America, New Haven, Yale University Press, 2003.

17. La constitution de Cadix définit en 1812 la nation comme la somme des communes, composées de vecinos. Cette figure permet de comprendre l'importance que les constitutions ibéroaméricaines accorderont, dans la définition de la nationalité, au système de jus soli combiné avec le domicile dans la définition de la nationalité. Cf. Tamar Harzog, Defining..., op. cit. Pour la définition de la nationalité, voir développement infra. 
rapide et pérenne qu'on peut constater au Rio de la Plata autour des droits des habitants, indépendamment de leur lieu de naissance ou de leurs origines. Il permet de perpétuer pendant presqu'un siècle la pratique de l'utilisation des indices sociaux - par exemple le témoignage d'un habitant voisin comme critère de la reconnaissance de ce statut ${ }^{18}$. Domingo F. Sarmiento, intellectuel et homme politique de premier ordre, qui consacrera les vingt dernières années de sa vie à dénoncer le manque d'attachement des étrangers envers l'Argentine, n'hésitera pas à défendre en même temps leurs droits civils, y compris la participation aux élections municipales sur la base des droits associés à la résidence ${ }^{19}$. Cette attitude apparemment contradictoire résume l'équation à laquelle étaient confrontées les élites politiques argentines : concilier le rattachement à la tradition juridique d'origine impériale - qui n'était pas exempte d'arrières pensées économiques et démographiques - avec la consolidation d'un État fondé sur le principe de la souveraineté nationale.

\section{LA LOI DE NATIONALité DE I 869 : ENJEUX LOCAUX, DESSEINS GLOBAUX}

La solution à cette équation devait passer pour beaucoup d'intellectuels et d'hommes politiques par l'adoption d'une règle propre à concilier le droit des habitants, fondé sur le domicile, avec la consolidation de la souveraineté nationale. La doctrine qui prévaut alors en Europe est celle du droit du sang, en rupture avec l'approche territoriale de l'allégeance, alors perçue comme signe de l'Ancien Régime ${ }^{20}$. En cohérence avec le droit du domicile, les États du Nouveau Monde vont quant à eux opter pour le principe du jus soli combiné avec le domicile (on peut acquérir la nationalité par naissance mais à condition que les parents étrangers soient domiciliés) et le jus sanguinis également combiné avec le domicile (les enfants des nationaux nés à l'étrangers acquièrent la nationalité de leurs parents à condition d'être domiciliés) ${ }^{21}$. Cette question prit rapidement une dimension internationale du fait des flux migratoires vers certaines régions (comme ce fut le cas pour l'Argentine) et des négociations des traités de paix et de reconnaissance que les différents États vont engager avec Madrid - l'Espagne ayant fait le choix des négociations bilatérales. Avec la question des indemnisations, celle de la nationalité

18. Dans les procédures de naturalisation, le témoignage d'un voisin suffisait pour attester une résidence. Ce n'est qu'en 1931 que le décret d'application de la loi de 1869 exigera un document de l'autorité policière.

19. Cf. Domingo F. Sarmiento, "La institución municipal», El Diario 14 de septiembre de 1887, in Domingo F. Sarmiento, Condición del extranjero..., op. cit., p. 313-320.

20. Pour la France, Cf. Patrick Weil, Qu'est-ce qu'un Français..., op. cit., p. 27-35; pour l'Amérique latine, $c f$. Estanislao $\mathrm{S}$. Zeballos, La nationalité au point de vue de la législation comparée du droit privé humain, Paris, Sirey, 1914, 2 tomes.

21. Cf. Estanislao S. Zeballos, La nationalité..., op. cit., p. 630-632. 
des péninsulaires restés sur le territoire américain constitue l'un des points importants des négociations ${ }^{22}$.

Dans le cas qui nous occupe, la Couronne d'Espagne réclamait que la paix soit scellée par la reconnaissance de la qualité d'Espagnol pour ceux qui n'avaient pas souhaité adhérer aux principes de la république indépendante, mais également pour les enfants nés après l'Indépendance sur le territoire de la République. Autrement dit, les prétentions espagnoles engageaient le projet de politique migratoire défini par la Constitution. La Confédération Argentine, qui avait voté le 7 octobre 1857 une loi de nationalité reconnaissant aux enfants d'étrangers le droit d'opter pour la nationalité de leurs parents, accéda aux demandes espagnoles et signa le traité "de reconnaissance, de paix et d'amitié» le 9 juillet $1859^{23}$. Mais la Confédération négocia en même temps les conditions d'intégration de l'État de Buenos Aires à la nation - la province avait fait sécession en 1852, et les exigences de Buenos Aires dans ce domaine étaient à l'opposé. En effet, le rapport de la commission d'examen de la Constitution rédigé par les représentants de Buenos Aires préconisait l'introduction du principe de jus soli et soulignait «les inconvénients qu'apporterait, pour un pays dont la population augmente principalement par l'immigration étrangère, la proclamation du principe de citoyenneté d'origine, qui en quelques années transformerait en étrangers une grande partie de ceux nés dans le pays ${ }^{24}$. L'amendement constitutionnel de 1860 introduit ainsi, à la demande de Buenos Aires, une référence explicite au principe de jus soli dans son article 67 , subordonnant la politique de nationalité aux objectifs populationnistes de la politique d'immigration.

Les Provinces, enfin unifiées, voient s'installer en 1862 le premier gouvernement national à Buenos Aires. Bartolomé Mitre, le président fraîchement élu, va aussitôt ordonner la renégociation de l'accord de reconnaissance avec l'Espagne dans le but d'annuler toute référence au principe de droit du sang que le gouvernement de la Confédération avait accepté d'introduire en 1859. Parallèlement, le juriste Valentín Alsina professeur de Droit naturel et des Gens à l'Université de Buenos Aires en 1834 avant que son opposition au régime de Rosas ne le mène avec bien d'autres libéraux à l'exil, présente en 1863 le premier projet de loi de nationalité destiné à préciser les conditions d'application du principe constitutionnel de jus soli. Or, dans celui-ci réapparaît curieusement la notion de filiation. Lors du long débat suscité par ce projet de loi, deux points de fond posent particulièrement problème. Le premier concerne la proposition d'introduire le jus sanguinis pour les enfants d'Argen-

22. Pour cette question, $c f$. Isidoro Ruiz Moreno, Relaciones Hispano-Argentinas. De la guerra a los tratados, Buenos Aires, Pellegrini e hijos, 1981.

23. Archives de la Chancellerie - Argentine, Section 8 "Traités et conférences ", boîte AH/0005. Le nouveau traité sera signé le 21 septembre de 1863 et supprimera les références à la loi de 1857.

24. Cf. Informe de la Comisión..., op. cit., p. 403. 
tins nés à l'étranger ${ }^{25}$. Certes, toutes les constitutions américaines reconnaissaient la nationalité des enfants de leurs ressortissants nés à l'étranger. Mais elles acceptaient en contrepartie que les enfants d'étrangers nés sur leur territoire puissent opter pour la nationalité de leurs parents. Or Buenos Aires s'était opposé à ce principe, jugé dangereux pour un pays d'immigration. La proposition était jugée par ailleurs malvenue par certains car à ce moment précis, la République Argentine négociait avec l'Espagne un amendement du traité de 1859 dans le but de supprimer toute référence au principe de filiation. Ce revirement paraît d'autant plus difficile à comprendre que Valentín Alsina avait participé à la convention qui avait recommandé en 1860 l'introduction du principe de jus soli dans la Constitution. Dans les interventions se glisse un argument qui semble avoir eu son poids dans la proposition de loi et qui montre combien les contingences peuvent se traduire en norme juridique. L'introduction du principe de filiation dans le projet de loi présenté en 1863 cherche en fait à apporter une solution au cas des enfants «martyrs de la République» nés pendant le long exil que connurent les opposants au régime de Juan M. de Rosas (1835-1852) dont Valentín Alsina faisait partie, et que le principe de jus soli risquait de transformer en étrangers ${ }^{26}$. Le problème majeur est alors soulevé par Guillermo Rawson, ministre de l'Intérieur, qui avait lui-même connu l'exil : sans la reconnaissance de la nationalité, la route vers la Présidence de la nation serait définitivement barrée pour leurs propres enfants ${ }^{27}$, une éventualité insoutenable pour ces notables qui nourrissaient pour leur progéniture les plus hautes ambitions politiques. L'article fut approuvé en première lecture par la chambre des députés avec l'exigence pour les fils d'Argentins nés à l'étranger de devoir exprimer leur volonté d'opter pour la nationalité devant un juge.

Le deuxième point qui fait débat en 1863 concerne le périmètre spatiotemporel d'application de la loi de nationalité, point qui était également sujet de négociation avec l'Espagne ${ }^{28}$. Pour le député Manuel Montes de Oca, l'article devait prendre le sens d'une déclaration de nationalité automatique pour la population se trouvant sur le territoire au moment de l'Indépendance, même s'ils étaient nés avant la révolution ${ }^{29}$. Les débats rendent compte des hésitations concernant les fondements de la nationalité. Selon Manuel Quintana député pour Buenos Aires et futur président de la République, c'est la

25. Cf. Congreso nacional, Diario de sesiones de la cámara de diputados, sesión $n^{\circ} 40$ del 19 de agosto -26 agosto de 1863 , p. 1-85.

26. Il s'agit de l'expérience de l'exil des opposants à Juan M. de Rosas qui avaient largement contribué à la chute de Rosas et qui se trouvaient maintenant au pouvoir. $C f$. intervention de José Benjamin Gorostiaga, idem, 19 août 1863, p. 16.

27. Cf. intervention de Guillermo Rawson lors de la séance du 24 août 1863, idem, p. 32. La constitution fixe en effet comme condition pour être candidat à la présidence, celle d'être argentin natif.

28. Le traité de reconnaissance paix et amitié de 1859 reconnaissait le droit de récupérer la nationalité espagnole à condition d'en faire la demande. L'Espagne avait obtenu la reconnaissance des mêmes droits du Chili, du Venezuela et de la Nouvelle Grenade.

29. Cf. Congreso nacional, Diario de sesiones, op. cit., p. 12. 
souveraineté originelle des Américains par rapport aux "envahisseurs» qui justifie la rétroactivité de la loi. Et il fait reposer son argumentaire sur la situation à laquelle le Mexique faisait face au même moment : l'illégitimité de l'occupation du Mexique par l'armée française était pour lui analogue à l'occupation de l'Amérique par les Conquistadors espagnols ${ }^{30}$. La réfutation incisive du député José Mármol, un autre compagnon de l'exil, met en évidence les apories du projet politique des formations post-impériales : «M. Quintana confond la conquête avec la colonisation. Nous n'avons pas été conquis. [...] Ce territoire a été colonisé par des hommes et des femmes espagnols et je ne connais aucun Argentin qui ait pour nom de famille Calfulcurá [...]. Alors je dis que jusqu'en 1810 nous étions espagnols car nous étions sous drapeau espagnol $\aleph^{31}$.

Malgré les longs débats et un vote favorable de la chambre basse, le projet de loi restera jusqu'en 1869 dans les tiroirs de la chambre haute, pendant que les négociations avec la Monarchie espagnole aboutissent en 1863 à un amendement du traité de paix signé avec la Confédération en 1859, et dans lequel disparaît la référence au jus sanguinis. Cet accord repose néanmoins sur une ambiguïté qu'aucune des deux parties n'a jugée utile d'éclaircir. Ainsi son article VII stipule-t-il qu'en matière de nationalité, chaque pays applique sa réglementation. Cela revenait à reconnaître de fait la double nationalité des enfants d'émigrants espagnols, équivoque qui sera source de tensions futures, notamment lorsque les fils nés en Argentine étaient appelés au service militaire en Espagne. Quant à la question de la nationalité de ceux nés avant l'indépendance, l'article du traité précise que la possibilité de récupérer la nationalité espagnole est offerte uniquement «aux Espagnols nés dans les actuels territoires de l'Espagne $»^{32}$. On considère alors que les habitants du territoire américain étaient argentins avant même l'Indépendance et on avance la date de la déclaration d'Indépendance de 1816 à 1810.

Une fois la réforme du traité de reconnaissance et d'amitié signée, le projet de loi de nationalité argentine est soumis à discussion au Sénat en 1869. Le texte est rapidement approuvé et promulgué le $1^{\text {er }}$ octobre 1869 , comme loi $346^{33}$. Il établit trois voies d'acquisition de la nationalité argentine : celle fondée sur le jus soli obligatoire - les enfants nés sur le sol argentin sont ainsi considérés comme argentins, indépendamment de leur volonté et de la nationalité et le domicile de leurs parents; celle fondée sur le jus sanguinis, qualifiée de "nationalité par option", permettant aux fils d'Argentins nés à

30. Cf. débat du 19 août 1863 , Idem, p. 23.

31. Idem, p. 23. Il s'agit mot pour mot des propos tenus par Juan Bautista Alberdi dans ses Bases..., op. cit., p. 60-61. Calfulcurá est le nom du chef indien de plusieurs tribus pehuelches qui habitaient un vaste territoire au sud de Buenos Aires.

32. Souligné dans le texte. $C f$. «Tratado de reconocimiento, paz y amistad... ", loc. cit., 1863.

33. D’après le député de Santa Fe Carlos Gómez, ce rapide consensus s'explique par le fait qu'en 1869 les enfants des exilés de la dictature de Rosas, particulièrement concernés par cet article, occupaient déjà des postes de pouvoir, faisant pression pour que l'article soit voté sans modification, cf. Congreso de la Nación, Cámara de Diputados, Diario de sesiones, sesión nº 12, 26/05/1913, p. 474. 
l'étranger d'accéder à la nationalité argentine sans exigence d'y être domiciliés. Quant à la naturalisation, elle concerne les étrangers de plus de 18 ans qui souhaitent devenir argentins après deux années continues de résidence. La nationalité peut être également acquise par mariage ou par services rendus à la République. La procédure doit s'effectuer auprès du juge fédéral de la circonscription du domicile du candidat ${ }^{34}$.

La loi de nationalité devient ainsi un instrument d'une politique migratoire qui associe les impératifs de peuplement fixés par la Constitution avec la possibilité de conserver la condition d'Argentin pour la première génération des enfants des nationaux nés à l'étranger. Comme l'a souligné Patrick Weil pour le cas de la France, l'opposition jus soli - jus sanguinis apparaît donc trop simplificatrice, et cet exemple montre que les États peuvent utiliser toutes sortes de combinaisons entre ces deux sources de nationalité pour répondre à des enjeux politiques qui ne sont pas toujours conciliables ${ }^{35}$. Dans ce cas précis, l'un des enjeux de la définition de la nationalité argentine, et non des moindres, est l'éventualité de signer un accord avec l'Espagne destiné à régler la question de la nationalité, concernant aussi bien la population d'avant la rupture que celle qui arrivait, après l'ordonnance de 1853, dans un cadre de libre émigration ${ }^{36}$. Mais le cas ici analysé montre également comment les notables locaux - autoproclamés «martyrs de la République» - peuvent développer leur propre stratégie dans les interstices des systèmes normatifs.

\section{Double visée Souverainiste de la politique de nationalité}

Ce dispositif juridique connut un succès incontestable, que l'on peut évaluer en nombres d'entrées sur le territoire national. Les raisons qui poussent les individus et les familles à migrer sont bien entendu multiples et ne sauraient se réduire à une politique d'accueil. Mais dans les prises de position publiques ou lors des discours proférés dans l'enceinte de l'Assemblée nationale, les hommes politiques attribuent volontiers l'accroissement des flux aux politiques qu'eux-mêmes encouragent et ceci explique pourquoi on attache autant d'importance à la modification du cadre légal.

L'Argentine est en tout cas la première destination latino-américaine des flux migratoires européens pendant la période des migrations de masse ${ }^{37}$. L'importance et l'intensité des flux ont néanmoins rapidement mis en évidence l'existence d'un «stock» d'étrangers que la libéralité de la loi de natio-

34. Cf. Congreso Nacional, Cámara de Diputados, Ley de Ciudadanía n 346, 1869, Buenos Aires, Imp. Del Siglo, 1874, p. 478-480.

35. Cf. Patrick Weil, Qu'est-ce qu'un Français?..., op. cit.

36. Cf. J. M. Pérez-Prendes y Muñoz-Arraco, El marco legal de la emigración española en el constitucionalismo, Colombres, Archivo de Indias, 1993.

37. Presque la moitié des migrants qui tentent l'aventure latino-américaine va choisir cette destination. Cf. Pilar González Bernaldo, "Flux migratoires», in Dictionnaire d'histoire de l'Amérique latine, Paris, Laffon, 2015 (sous-presse). 
nalité n'avait pas réussi à «diluer». En 1874, ils représentent déjà $14 \%$ de la population totale, ils sont $28 \%$ en 1888 et atteignent $30 \%$ au moment du déclenchement de la Grande Guerre ${ }^{38}$. Autrement dit, plus d'un habitant sur quatre est alors juridiquement étranger. Les chiffres sont encore plus spectaculaires dans la ville de Buenos Aires. En 1909 45\% de sa population et $67 \%$ de la population masculine en âge de voter (entre 20 et 60 ans) est née à l'étranger. En 1914 c'est un habitant sur deux qui est né à l'étranger, tout âges et sexes confondus.

La présence massive d'étrangers dans la force de l'âge commence à inquiéter sérieusement les hommes politiques et l'opinion publique à partir de 1880. Et les regards se portent rapidement sur la loi de nationalité votée en 1869, jugée inapte à répondre à ce problème. À partir de cette date et jusqu'à la publication en 1931 du décret d'application de la loi de 1869, l'activité législative autour des différents projets de réforme de la loi est aussi continue qu'infructueuse. J'ai pu répertorier pendant toute la période 26 projets qui impliquaient directement une réforme de la loi de nationalité présentée à la discussion. Un seul projet de loi sera finalement sanctionné en 1917 et il s'agit d'un amendement mineur à la procédure de naturalisation fixée par la loi de $1869^{39}$.

\section{Graphique $n^{\circ} 1$}

Activité parlementaire autour du projet de loi sur la nationalité (1862-1931)

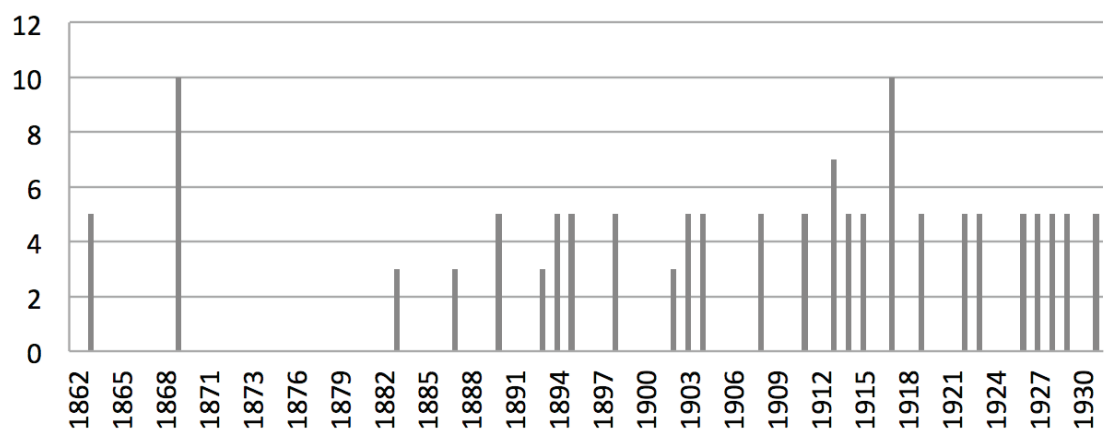

Valeurs de la série : 10 projets sanctionnés; 7 projets acceptés par la chambre des députés et réceptionnés par la chambre haute. 5 projets examinés par la chambre des députés; 3 projets de loi examinés par la chambre des députés et qui touchent en partie la question de la citoyenneté.

Bien que presque toutes les tentatives de modifier la loi aient échoué, il n'est pas sans intérêt de les analyser car elles permettent de dégager les points

38. Calculs de Blanca Sánchez-Alonso, 'Making sens of immigration policy: Argentine, 18701930', The Economic History Review, 66, 2, (2013), p. 601-627.

39. Il s'agit de la loi 10.256 du 18/09/1917, $c f$. Congreso de la Nación, Cámara de Diputados, Diario de sesiones, 1917. 
sur lesquels il a été impossible de trouver un consensus ainsi que les enjeux qui entourent ces débats.

Les différentes propositions de réforme peuvent se classer en quatre types : les projets exigeant une demande individuelle comme condition de naturalisation, ceux qui introduisent d'autres conditions supplémentaires, ceux destinés à inciter à la naturalisation pour faciliter aux nationaux l'accès à certains postes et enfin, la simple naturalisation automatique.

Graphique $n^{\circ} 2$

Projets de loi de nationalité : entre libre demande, incitation et imposition 1868-1931

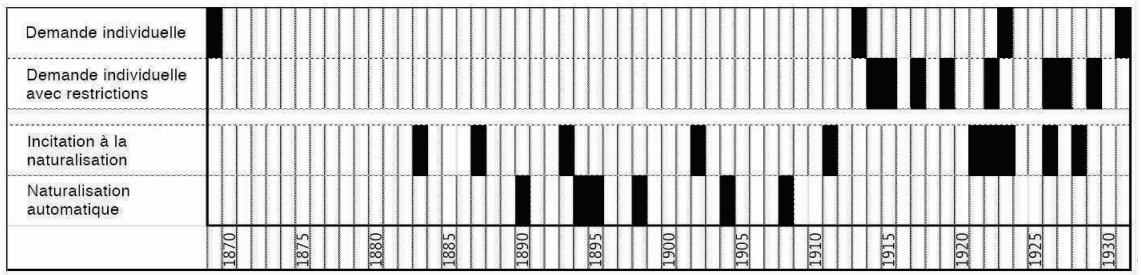

Deux périodes bien distinctes se dégagent. Une première qui va de 1883 jusqu'en 1912, pendant laquelle tous les projets présentés concernent soit la naturalisation automatique, soit des mesures destinées à inciter à la naturalisation volontaire, avec néanmoins des différences significatives concernant les années de résidence requises pour bénéficier de la loi - cela peut aller de deux à dix ans de résidence, condition associée à d'autres critères comme le mariage avec un national, une descendance argentine, être propriétaire, etc. Tous ces projets introduisent une clause permettant de refuser la naturalisation afin de ne pas se heurter à l'article 20 de la constitution qui établit que les étrangers ne peuvent pas être forcés à acquérir la nationalité. La deuxième période correspond à celle ouverte à l'échelle nationale par la réforme électorale de 1912 et le déclenchement de la Grande Guerre, jusqu'au choc provoqué par le krach boursier de 1929. L'idée d'une naturalisation automatique est alors définitivement abandonnée et les projets cherchent plutôt à préciser le cadre d'application des principes formulés dans la loi de 1869.

Si les données du graphique tracent une périodisation assez nette, la question demeure de savoir quelles sont les raisons qui poussent les hommes politiques, pourtant issus de différentes formations - conservateurs, radicaux et socialistes - à proposer entre 1883 et 1912 plusieurs projets de loi destinés à introduire la naturalisation automatique. En règle générale en effet, elle est plutôt utilisée pour définir la nationalité dans le cas de l'incorporation de nouveaux territoires suite à une annexion ou une conquête, que pour définir le statut juridique d'une population étrangère arrivée de son propre chef pour travailler sur le territoire d'un autre État souverain. Les auteurs de ces projets partent tous du même constat à propos du faible taux de naturalisation des étrangers, mais l'expliquent par des causes différentes : alors que 
pour les conservateurs, c'est le produit de l'indifférence des étrangers envers le pays d'accueil qui les a pourtant si généreusement accueillis, les radicaux mettent l'accent sur les attaches affectives des étrangers envers leur nation d'origine qu'il faut les inciter à couper, alors que les socialistes dénoncent plutôt les obstacles techniques qui entravent volontairement l'application de la loi. Mais tous se rejoignent dans l'idée qu'il faut apporter une solution légale et collective à une situation qu'ils considèrent comme alarmante pour la nation argentine ${ }^{40}$.

Aux dimensions de politique intérieure s'ajoutent les répercussions de cette présence étrangère dans la sphère internationale. Tout d'abord par les tensions multiples que les conflits de loi alimentent avec les États d'origine de ces populations concernant aussi bien les migrants que leur descendance. Ces désaccords reposent sur le double différend entre le principe de jus soli argentin et le jus sanguinis adopté par de nombreuses nations européennes et le choix fait par l'Argentine d'appliquer la loi du domicile alors que les nations européennes, en accord avec l'esprit du code napoléonien, considèrent que lorsqu'il y a conflits des lois, c'est celle de la nation d'origine du plaignant qui doit s'appliquer ${ }^{41}$. À cela vient s'ajouter un contexte international qui rend particulièrement dangereuse la présence des «colonies étrangères» sur le territoire national. La représentation du "colon" change alors sensiblement. Elle ne renvoie plus systématiquement à une population composée de familles d'agriculteurs venues labourer la terre, peupler et développer des territoires gagnés à la "civilisation» et contribuer ainsi à la consolidation de la souveraineté argentine sur ces territoires. On attire de plus en plus l'attention sur le danger que comporte la présence de "colonies étrangères", installées dorénavant en ville et organisées à travers un dense réseau associatif, disposant de leurs organes de presse, d'écoles et d'hôpitaux communautaires. Une nation sans nationaux, résume Domingo F. Sarmiento désabuséf ${ }^{42}$.

Le danger principal ne réside pas cependant, comme le suggère l'écrivain nationaliste et historien de la littérature Ricardo Rojas dans le prologue à la réédition des écrits de Domingo F. Sarmiento en 1928, dans le "chaos cosmopolite» que les étrangers représentent pour l'homogénéité de la nation, mais plutôt, comme le souligne sans cesse ce dernier, dans le péril qu'ils constituent pour la souveraineté nationale ${ }^{43}$. Il ne s'agit pas d'un repli identitaire mais

40. Cf. Diario de Sesiones de la Honorable Cámara de Diputados y de Senadores, Buenos Aires, Honorable Congreso de la Nación; consultés entre 1862 et 1931.

41. Cf. Estanislao Zeballos, Las conferencias en Williamstown. Buenos Aires, Talleres Gráficos de la Penitenciaría Nacional, 1927, p. 70. Pour ces questions, voir Philippe Rygiel, Une impossible tâche? L'institut de droit international et la régulation des migrations internationales, 1879-1920, Thèse d'habilitation à diriger des recherches, Université Paris 1 Panthéon-Sorbonne, Paris I, 2011, p. 108166.

42. Cf. Domingo F. Sarmiento, «Una nación sin nacionales» et «Los Italianos en las escuelas de Buenos Aires y Montevideo ", in La condición del extranjero..., op. cit., p. 467-479.

43. C’est mon principal point de désaccord avec la thèse de Lilia Ana Bertoni, $c f$. Pilar González Bernaldo, "Compte-rendu de l'ouvrage de Bertoni L., Patriotas, cosmopolitas y nacionalistas. La construcción de la nacionalidad argentina a fines del siglo XIX», Annales. E.S.C, 2003/1, p. 216-218. 
d'un souci souverainiste qui anime les débats des années 1890 et qu'on doit placer dans le contexte de l'expansionnisme européen qui inquiète alors particulièrement les élites argentines. Il autorise des doutes raisonnables concernant les desseins inavoués des États d'origine des immigrants provenant des nations européennes, prises de "fièvre impériale »44. Dans l'indifférence à peu près générale, Domingo F. Sarmiento attire l'attention sur ce péril depuis les années $1850^{45}$. Le danger devient beaucoup moins spéculatif lorsque le sénateur du royaume d'Italie, Girolano Boccardo, laisse entendre en 1885 que compte tenu de l'importance d'une communauté d'ancrage dans le Rio de la Plata, cette région pouvait devenir une province italienne ${ }^{46}$. Avant même ces déclarations, Domingo F. Sarmiento avait déjà jugé utile de reproduire en 1882 un article de La Revue Politique et Littéraire destiné à dénoncer, dans un contexte postérieur à la défaite française contre la Prusse, le dessein impérial de la politique de colonisation allemande en Amérique méridionale et en Afrique : «L'esprit d'entreprise sera tel que dans un temps donné les centres colonisés seront devenus en fait des provinces allemandes ${ }^{47}$. Il fait siennes les craintes françaises et soupçonne le gouvernement italien des mêmes intentions impériales. Après tout «si les Anglais l'ont fait pour s'emparer des Iles Malouines [1833], pourquoi l'Italie ne le ferait-elle pas?» Il en conclut que les pays d'Amérique risquent de devenir des colonies italiennes, espagnoles ou allemandes ${ }^{48}$. Les exemples qui donnaient une certaine crédibilité à ces craintes ne manquaient pas, aussi bien au Mexique où le peuplement du Texas par des colons nord-américains avait abouti d'abord à l'annexion de ce territoire par les États de l'Union en 1845 et ensuite à la guerre contre les États-Unis qui se solda par la perte de presque la moitié du territoire mexicain en 1848. A peine une décennie plus tard la France occupait militairement le Mexique, fait qui, comme nous avons pu le constater, n'avait pas laissé indifférents des hommes politiques des contrées rioplatenses. En 1862, l'Espagne reprenait possession de la République Dominicaine et menaçait d'occuper le Pérou en 1866. Mêmes les exploits des aventuriers pouvaient comporter un danger pour la souveraineté territoriale, comme en témoignait l'aventure de William Walker au Nicaragua. Avec plus d'imagination le Français Antoine de Tounens se faisait proclamer dans les années 1860 roi d'Araucanie et tentait pendant une décennie de faire valoir ses droits de souverain sur la Patagonie, territoire que les autorités de Buenos Aires consi-

44. Cf. Frederic Cooper, Le colonialisme en question. Théorie, connaissance, histoire, Paris, Payot, (1 $1^{\text {re }}$ édition 2005), 2010.

45. Cf. Domingo F. Sarmiento, "Las colonias libres del Plata», in La condición del extranjero..., op. cit., p. 158.

46. Cf. Lilia Ana Bertoni, Patriotas, cosmopolitas y nacionalistas. La construcción de la nacionalidad argentina a fines del siglo XIX, Buenos Aires, FCE, 2001, p. 103; Mark I. Choate, 'From Africa to America', Emigrant Nation. The making of Italy Abroad, London/Cambridge, Harvard University Press, 2008, p. 21-56.

47. Cf. Domingo F. Sarmiento, "Ojo al Cristo, que es la Plata», El Nacional, 12 juillet 1882, in Condición del extranjero en América..., loc. cit., p. 192-195.

48. Ibidem. 
déraient avoir hérité de la Couronne espagnole, et qu'elles n'hésitèrent pas à incorporer ensuite à travers plusieurs campagnes militaires qui décimèrent la population Indienne. À la fin du XIX ${ }^{\mathrm{e}}$ siècle, la carte du monde se redessinait et les Argentins ne restaient pas indifférents à l'"orgie impérialiste» qui embrasait alors l'Europe ${ }^{49}$. Le gouvernement choisit le grand spécialiste de droit international public et consul argentin à Berlin Carlos Calvo, pour représenter l'Argentine à la Conférence de Berlin de $1884^{50}$. Il n'est donc pas tout à fait surprenant d'entendre en 1908 Lucas Ayarragaray, député de la province d'Entre Rios, défendre son projet d'une naturalisation automatique en alertant l'assemblée sur la situation de l'Afrique du Sud, théâtre de la guerre des Boers. L'exemple donne à réfléchir : comme Lucas Ayarragaray n'hésite pas à le rappeler, les colonies britanniques installées sur ce territoire sont une pièce centrale du déclenchement de cette guerre ${ }^{51}$.

La réforme de la loi électorale de 1912 et l'éclatement de la Grande Guerre semblent changer la donne. Le graphique $\mathrm{n}^{\circ} 2$ montre que ce nouveau contexte coïncide avec l'abandon de l'idée d'introduire une loi de naturalisation automatique comme réponse à la forte présence des étrangers sur le territoire de la république ${ }^{52}$. En effet, à partir de 1913, les différents projets de nationalité présentés reprendront le principe d'une naturalisation individuelle et impliqueront la demande explicite du candidat. Le premier d'entre eux est présenté par le député de Santa Fe Carlos Gómez en août 1913. Son projet cherche moins à "diluer" le stock d'étrangers qu'à réguler "l'alluvion électorale» en contrôlant l'accès de nouveaux arrivants -dont les fidélités politiques étaient incertaines, surtout pour les conservateurs dans le corps électoral ${ }^{53}$. Il ne s'agit plus de protéger la souveraineté territoriale par une naturalisation automatique des ressortissants des puissances européennes mais de réguler l'accès des étrangers à la communauté des citoyens.

Ce changement n'est peut-être pas étranger à la diversification des flux migratoires depuis la fin du XIXe siècle, lorsque l'émigration vers l'Amérique devient une réponse possible pour de nouvelles populations prises en tenaille par les conflits en Europe, phénomène que la mise en place d'une législation migratoire restrictive aux États-Unis va intensifier. C'est alors qu'on voit apparaitre les premières références explicites aux restrictions culturelles,

49. Selon l'expression de Christopher A. Bayly, Naissance du monde moderne (1780-1914), Paris, Éditions de l'Atelier, 2007, p. 374-383.

50. Cf. Victor Cutolo, Nuevo diccionario biográfico argentino, Buenos Aires, 1969, T. II, p. 56-57; Philippe Rygiel, Une impossible tâche..., op. cit., p. 32.

51. Diario de sesiones de la cámara de diputados, 1908, p. 479.

52. À l'exception près du projet présenté par le député Daniel Talens en 1928 de naturalisation automatique mais uniquement pour les étrangers arrivés en bas âge et ayant été scolarisés en Argentine, cf. "Proyecto de naturalización de extranjeros del diputado Talens", in Congreso de la nación, Diario de sesiones de la cámara de diputados, 12 juillet 1928, p. 195.

53. Son projet destiné à fermer l'accès à la nationalité est proposé cinq mois après la tenue des premières élections qui suivirent la réforme électorale, faisant rentrer 5 représentants du parti socialiste au Congrès de la nation. Cf. Congreso Nacional, Diario de sesiones de la cámara de diputados, reunión $\mathrm{n}^{\circ} 12$ del 26 de mayo de 1913, p. 469-487. 
morales, idéologiques, intellectuelles et politiques à l'accès à la nationalités ${ }^{4}$. Mais comme pour la période antérieure, ces projets ne seront pas sanctionnés. Ils vont néanmoins inspirer le décret d'application de la loi de 1869 qui sera finalement promulgué en 1931 et qui va réguler l'accès à la nationalité à partir de critères qui s'inscrivent dans un autre dessein, celui de la préservation de l'identité nationale.

\section{Conclusion}

Le cas ici analysé vient sans aucun doute conforter la thèse selon laquelle la politique de la nationalité fait partie des instruments utilisés pour mettre en place une politique d'immigration. Il témoigne de la convergence de différents dispositifs qui dans ce cas précis sont redoublés par une extraordinaire pérennité de la tradition juridico-législative hispanique fondée sur la figure de l'habitant comme sujet de droit, dissociant la nationalité de la citoyenneté. En Argentine la politique d'immigration comme la politique de nationalité répondent à l'injonction alberdinienne de "peupler» le pays. Cela explique sans doute le solide attachement de la nation argentine au jus soli obligatoire, qu'elle n'a pas démenti tout au long de son histoire postérieure à l'indépendance. Mais la convergence ne présuppose pas la cohérence entre ces différentes logiques. D'une part, la solide tradition du jus soli n'a pas empêché l'introduction du jus sanguinis, principe qui a connu également une remarquable pérennité historique malgré son irrécusable caractère anticonstitutionnel. Le statut de l'habitant garanti par la constitution a, d'autre part, pu jouer un rôle dissuasif pour la naturalisation, dans la mesure où il permettait l'exercice de la citoyenneté dans sa dimension locale et sociale, voire même nationale sur certains aspects. Mais comme ce travail le suggère, la politique de nationalité envisagée cherchait dans un premier temps moins à élargir les droits politiques des habitants qu'à confirmer la souveraineté de l'État argentin sur son territoire. Le nouveau contexte international ainsi que les enjeux géopolitiques dans lesquels cette politique s'inscrit permettent de mieux comprendre les projets de réforme et les débats. Ainsi, autant lors de la discussion de la loi de nationalité adoptée en 1869 que lors des débats que suscitent les différents projets de réforme, les hommes politiques cherchèrent à apporter des réponses aux besoins de consolidation de la souveraineté territoriale dans le contexte d'intensification des flux d'immigrants et de regain de l'expansion impériale européenne. Malgré la fébrilité législative, les outils

54. Ainsi le projet de Carlos Gómez établit dans son article 13 la liste des exclus de la naturalisation : ceux qui ne maitrisent pas la langue espagnole, ceux dont les lois interdisent l'entrée sur le territoire national, les étrangers provenant des pays en guerre contre la république, les anarchistes et les impliqués dans la traite des blanches, les handicapés mentaux, les sourds et muets, les condamnés, les individus mêlés à une faillite frauduleuse et tous ceux qui ne peuvent témoigner de leur bonne conduite. 
avec lesquels ils y ont fait face ne seront pas modifiés et reposent sur une tradition juridique impériale que l'État national reprendra à son compte pour attirer de nouveaux «habitants».

Le cas argentin n'est certainement pas "représentatif» au sens statistique du terme. À plusieurs égards il semble plutôt exceptionnel, aussi bien en ce qui concerne le choix d'un jus soli absolu (il sera le seul État des Amériques à l'établir) qu'en termes de précocité des droits accordés aux étrangers. Mais comme l'avait suggéré Carlo Ginzburg il y a plus de trente ans, un cas limite peut se révéler représentatif dans la mesure où il permet de circonscrire les possibilités latentes d'une situation ${ }^{55}$. Il met dans ce cas en évidence la nécessité d'élargir le contexte spatio-temporel pour comprendre les logiques qui sont à l'œuvre, où le plus global - insérer le nouvel État dans un système d'interactions multiples tout en faisant respecter internationalement sa souveraineté territoriale - s'articule avec le plus local - le jeu d'alliances entre notables, destiné à garantir le pouvoir localement.

Pilar González Bernaldo de Quirós est Professeur d'histoire et de civilisation de l'Amérique latine à l'Université Paris Diderot 